Infusionstherapie 1987;14:295-298

\title{
Jahres-Inhalt, Vol. 14, 1987
}

Abstrakts zur 6. Gemeinsamen Jahrestagung der Deutschen Arbeitsgemeinschaft für künstliche Ernährung (DAKE) und der Österreichischen Arbeitsgemeinschaft für klinische Ernährung (AKE), 26.-29. März 1987, Augsburg 4 Informationen für die Klinik 22 Hayungs, J., Düsseldorf, et al. Der Einfluß von Lipidemulsionen auf die Stickstoffbilanz im Rahmen eines perioperativen parenteralen Ernährungsregimes 23

Buchbesprechungen 37

Panteliadis, Ch.; Kremenopoulos, G.; Soumpase, V. und Avgoustidou, P., Thessaloniki/Griechenland

Erfahrungen mit MCT-haltigen Fettemulsionen bei Frühund Neugeborenen $\quad 38$ Empfehlungen zur parenteralen Infusions- und Ernäh rungstherapie im Kindesalter 41

Fauth, U.; Heinrichs, W. und Halmágyi, M., Mainz Stoffwechselmodelle zur Interpretation indirekt kalorischer Messungen bei Intensivpatienten $\quad 48$ Informationen für die Klinik 60

Lünstedt, B.; Deltz, E.; Kähler, M. und Bruhns, A., Kiel Randomisierte Studie zum Vergleich zwischen langketti-gen (LCT) und mittelkettigen (MCT) Triglyzeriden als Kalorienträger in der postoperativen Ernährungstherapie 61

Park, W.; Paust, H.; Brösike, H.; Knoblach, G.;

Mesche, M. und Helge, H., Berlin

Einfluß der Kohlenhydratzufuhr auf die Leuzinoxydation bei Neugeborenen. - Untersuchungen mit dem 13C-Leuzin-Atemtest 66

Helbig, J., Tutzíng

Bericht über das 9. Hohenheimer Magnesium Sympo sium 71

Pick, J.; Adelt, Th.; Huse, K. und Bock, W.J., Düsseldorf

Liquor- und Plasmaaminogramme bei Patienten mit hirneigenen und hirnfremden Tumoren des ZNS 73

Sonderbände 77

Weidler, B.; v. Bormann, B.; Lohmann, E. und Elmadfa, I., Gíeßen; Peil, J. und Schwanen, N., 
Bad Nauheim; Sommermeyer, K., Bad Homburg

Über den Einfluß von Teilchengröße und Emulgator auf

pharmakokinetische Kenndaten einer parenteral appli-

zierten Fettemulsion 78

Editorial 92

Georgieff, M., Boston, Mass. I USA

Intravenöse Ernährung - Möglichkeiten und Grenzen der

Anwendung von Glukose und Xylit nach Trauma und

Sepsis unter besonderer Berücksichtigung des Leberstoff-

wechsels

93

Förster, H., Frankfurt

Fruktose und Sorbit als energieliefernde Substrate für die

parenterale Ernährung 98

Informationen für die Klinik 110

Moldawer, L.L., Göteborg/Schweden,

Georgieff, M., Boston/USA,

Ekman, L. und Wretlind, A., Stockholm/Schweden

Die metabolische Reaktion auf Glyzerol während paren-

teraler Ernährung IIl

Reinauer, H., Düsseldorf

Grenzen der extensiven Verwendung von Glukose als

Infusionskohlenhydrat in der parenteralen Ernährung . . 116

Ahnefeld, F. W.; Grünert, A. und Schmitz, J- E., Ulm; Bäßler, K. H. und Halmágyi, M. , Mainz;

Mehnert, H., München

Kohlenhydratintoleranzen als Gefahr bei der Infusions

therapie 124

Buchbesprechungen 130

Bekanntmachung und Hinweis über Kohlenhydratintole

ranzen 131

Editorial 136

Fürst, P.; Stehle, P. und Graser, T.A., Stuttgart

Fortschritte in der Aminosäurenanalytik unter beson

derer Berücksichtigung der Ermittlung intrazellulärer

Aminosäurenmuster 137

Roth, E. und Karner, J., Wien

Intrazelluläre Aminosäurenkonzentrationen bei verschie-

denen Krankheitszuständen 147

Staedt, U.; Holm, E.; Leweling, H.; Jacob, S.

und Striebel, J.-P., Mannheim

Intrazelluläre Aminosäurenkonzentrationen der Musku-

latur dreier Tierspezies unter physiologischen, pathophy-

siologischen und infusionstherapeutischen Bedingungen . 151

Informationen für die Klinik 159

Bratusch-Marrain, P. R. und Gasic, S., Wien

Lebervenenkatheter-Technik: Methode zur Erfassung 
metabolischer und hormoneller Variablen im Splanchnikusgebiet $\quad 160$

Wicklmayr, M.; Rett, K.; Dietze, G.

und Mehnert, H., München

Erfassung des Substratstoffwechsels der Skelettmuskulatur beim Menschen 164

296

Jahres-Inhalt 1987

Karner, J. und Roth, E., Wien

Blutflußmessung und Kanülierungstechnik im akuten und chronischen Hundemodell 170

Häussinger, D., Freiburg

Isoliert perfundierte Rattenleber: Ein experimentelles

Modell für Untersuchungen zum Ammoniak- und Ami-

nosäurenstoffwechsel 174

Schadewaldt, P., Düsseldorf

Das isoliert perfundierte Hinterbein der Ratte. - Metho

dik und Anwendung 179

Erratum 184

Sonderbände 184

Hartmann, F; Plauth, M.; Viellard-Baron, D.

und Bauder, D., Tubingen

Isolierte Perfusion des Rattendünndarms mit Perfluoro-

tributylamin als künstlichem Sauerstoffträger

Rett, K.; Maerker, E.; Wicklmayr, M.; Dietze, G.

und Mehnert, H., München

Die Methodik des isoliert-perfundierten Rattenherzens

nach Langendorff in der Ernährungsforschung

Palacios Rubio, V.; Sanz Gonzalo, T.;

Montón Dito, J. M.; Garcia Jalón, A. und

Calvo Ruata, M. L., ZaragozalSpanien

Kalorienzufuhr in der parenteralen Ernährung während der Streßphase 196

Culebras-Fernandez, J- M,; de la Hoz Riesco, M.;

Villares Garcia, C; Hurtado Fernandez-Llamazares, G. und Alonso Villalba, A., LeónlSpanien

Verbesserung des Ernährungszustands durch hyperkalorische periphere parenterale Ernährung (HPPN) in der frühen postoperativen Periode nach elektiver Bauchchirurgie 202

Rausch, W.-D.; Schnecker, K. und

Bruck, J., WienlÖsterreich; Riederer, P., Würzburg

Veränderungen organischer Säuren in Plasma und Liquor bei Hirninfarkt209

Brenner, U.; Müíler, J. M.; Keller, H. W.; Walter, M. 
und Thul, P., Köln

Der Vergleich prognostischer Ernährungsindizes zur

präoperativen Erfassung von Risikopatienten. - Eine

prospektive Prüfung 215

Wiedeck, H., Ulin

Untersuchungen zur Homöostase der Plasmaaminosäu-

ren unter standardisierter parenteraler Ernährungsthera-

pie bei akut nekrotisierender Pankreatitis 224

Informationen für die Klinik 228

Scheffler, P.; Jung, F.; Mrowietz, C,; Waldhausen, P. und Leipnitz, G., Homburg; Häuser, B., München Hämorheologische, mikro- und makrozirkulatorische Ef-fekte einer hypervolämischen Infusion mittelmolekularer Hydroxyäthylstärke $(10 \%, 200000 / 0,62)$ bei Gesunden. . 233

Aprili, Z.; Hauser, R.; Norlindh, T. und

Kahnemouyi, H., WinterthurlSchweiz

Vorbeugung von Fettleber unter indirekt-kalorimetrisch

gesteuerter totaler parenteraler Ernährung 239

Sonderbände 244

Brenner, U., Köln; Herbertz,L., Düsseldorf; Meibert,M.,

Müller, J.M., Köln; Reinauer, H, Düsseldorf

Der Einfluß des Dünndarms auf den 3-Methylhistidin-

Stoffwechsel des Menschen 248

Heinrichs, W.; Fauth, U.; Seufert, W. und Halmágyi, M.,

Mainz

Auswirkung einer durch L-Lysin ausgelösten Aminosäu-

ren-Imbalanz auf die Stickstoffbilanz bei vollständig par-

enteral ernährten Patienten 252

Metzner, Ch.; Hartig, W. und Matkowitz, R-, Leipzig; Graubaum, H.-J.; Jung, K. und

Wagenknecht, C, Berlin; Ritter, R., Leipzig

Wertigkeit verschiedener Aminosäurengemische für die

Behandlung des chronischen Leberschadens 256

Neuhäuser-Berthold, M.; Böhler, S.; Berthold, D. und Bässler, K.H., Mainz

Infusionslösung mit hohem Anteil an verzweigtkettigen

Aminosäuren (VKAS) und hochprozentige Aminosäu-

renlösung im experimentellen Tiermodell 262

Zwiauer, K. und Widhalm, K., Wien

Besonderheiten der perioperativen Ernährungstherapie

bei Kindern 267

Erratum 272

Semler, P., Berlin; Sommermeyer, K., Oberursel

Bedeutung und Prüfung der Kompatibilität von Misch-

lösungen mit Fettemulsionskomponente für die parente

rale Ernährung 274

Grundmann, R. und Tuber gen, D., Köln

Humanalbumintherapie und prognostischer Wert der kol-

loidosmotischen Druckbestimmung auf der chirurgischen

Intensivstation 284 
Informationen für die Klinik 289

Gramm, H.-J.; Baudisch, H.; Schlagenhaufer, S.;

Goecke, J. und Frucht, U., Berlin

Spurenelementgehalt in Lösungen zur parenteralen Er

nährung und Blutderivaten 290

Jahres-Inhalt $1987 \quad 295$

Autorenverzeichnis 1987

299

Buchbesprechungen 300

Jahres-Inhalt 1987

297

Supplement 1, Februar 1987

Böhles, H., Eriangen

Cholestase bei totaler parenteraler Ernährung (TPE). -

Eine Übersicht 3

Dölp, R., Fulda, Grünert, A.; Schmitz, E.

und Ahnefeld, F. W. , Ulm

Klinische Untersuchungen zur periphervenösen parenteralen Ernährung. - Einfluß einer 3,5\%igen Aminosäurenlösung auf den postoperativen Stoffwechsel unter besonderer Berücksichtigung des Aminosäurenhaushalts . . 10

Sonderbände 16

Schmoz, G.; Hartig, W.; Brunner, H.-P.

und Weiner, V., Leipzig/DDR; Erhard, V.

und Vetter, K., PotsdamlRehbrückelDDR

Erfassung der Gefährdungslage mit Hilfe ernährungsdiagnostischer Untersuchungen bei chirurgischen Patienten. - 1. Mitteilung: Bestimmung der präoperativen $\mathrm{Ge}$ fährdungslage bei Patienten mit Magenkarzinom 17

Weidler, B.; v. Bormann, B.; Lohmann, E.;

Muhrer, K. H. und Hempelmann, G., Gießen,

Prinzler, H.-J., Braunfels

Über den parenteralen Einsatz einer Fettemulsion in der frühen postoperativen Phase 28

Kleinberger, G.; Druml, W.; Laggner, A.

und Lenz, K. , Wien/Österreich, Heinzel, G. , Biberach Bestimmung der Aminosäurenkinetik im Plasma durch eine Bolus-Infusionskinetik 40

Cavallé Obíols, ¥.; Monterde Junyent, J.;

Schwarts Riera, S. und Farriol Gil, M.,

Barcelona!Spanien

Die Anwendung der Peritonealdialyse zur Nährstoff-

zufuhr 46

Georgieff, M.; Geiger, K. und Lutz, H., Mannheim;

Moldawer, L.L., GothenburglSchweden; Wagner, D.;

Blackburn, G.L. und Bistrian, B.R., Boston/USA, 
Fekl, W., Erlangen

Stoffwechselorientierte postoperative Ernährungstherapie - Möglichkeiten und Grenzen der Anwendung von

Glukose und Xylit 53

Supplement 2, April 1987

Schönitzer, D., Innsbruck/Österreich

Die Stellung der Kell-Inkompatibilität im Rückblick auf

15 Jahre Mutter-Kind-Paß-Serologie 3

Panzer, S.; Maier, F; Höcker, P.; Mayr, W.R.

und Hinterberger, W., Wien/Österreich

Thrombozytentransfusion: Plättchenanstieg in Abhängigkeit von klinischen und immunologischen Voraussetzun-

gen 10

Glück, D.; Koemei; K.; Stampe, D.

und Kubanek, B., Ulm

Gewinnung von Thrombozytenkonzentraten und gerin-

nungsaktivem Frischplasma mit dem System Haemo-

netics PCS 16

Laser, J. und Krüger, J., Köln

Einfluß von wiederholten Leukozytapheresen auf das

Lymphozytogramm von Blutspendern

23

Sonderbände 26

Borchardt, W.; Heinzow, B. und Ziegler, A., Kiel

Bindung von Pharmaka an künstliche Plasmaersatzmittel. 28

Hopf, H.-B. und Siepmann, H.P., Dormagen

Vergleichende Untersuchung zur Beeinflussung von Blut,

Blutgerinnung, Herz und Kreislauf durch 3\%ige modifi-

zierte, flüssige Gelatine und 6\%ige niedermolekulare

Hydroxyäthylstärke 31

Klose, R., Ludwigshafen, Feldmann, U.

und Hoecker, P., Mannheim

Der Einfluß von 1O\%iger Hydroxyäthylstärke 200/0,5

und 10\%igem Dextran40 auf Fließeigenschaft und Gerinnung von Vollblut in vivo 37

Lenz, G.; Sugg, U.; Peter, E. und Mayer, G., Tubingen

Positive Minor-Kreuzprobe durch erythrozytäre T-Akti-

vierung bei einem Säugling mit Kolonperforation 43

Supplement 3, September 1987

Editorial 3

Ekman, L. und Wretlind, A., Stockholm/Schweden;

Moldawer, L., GöteborglSchweden

Neue Entwicklungen bei Fettemulsionen in der parente

ralen Ernährung

4

Grünert, A., Ulm

Fettemulsionen: Grundlagen pathophysiologischer Ein-

flüsse und klinischer Applikationen 10 
Sonderbände 18

Wolfram, G., München

Essentielle Fettsäuren in der parenteralen Ernährung. . . 20

Carpentier, Y.A.; Richelle, M.; Bihain, B.E.;

Dahlan, W. und Haumont, D., BrüssellBelgien;

Deckelbaum, R.J., New York/USA

Interaktionen zwischen exogenem Fett und Plasma/Lipo-

proteinen 29

Böhles, H., Erlangen

Die Stellung von Carnitin in der klinischen Ernährung. -

Ein Erklärungsversuch 33

Eckart, J.; Neeser, G. und Adolph, M., Augsburg;

Hailer, S. und Wolfram, G., München

Klinische Untersuchungen mit LCT- und MCT-haltigen

Fettemulsionen

38

298

Jahres-Inhalt 1987

Meng, H.C., Nashville, Term./USA

Die Bedeutung von Eicosapentaensäure (EPA) in der

Zusatzernährung 51

Supplement 4, September 1987

Nydegger, U.E., BerniSchweiz; Vaudaux, P.,

GenflSchweiz; Castelli, D., Lugano/Schweiz

Entwicklungen in der Hämapherese 1986. - Aktueller

Bericht über die 4. Jahresversammlung der Europäischen

Gesellschaft für Hämapherese 4

Kretschmer, V. etal., Marburg

Nebenwirkungen und technische Probleme bei Zyt-

apheresen mit Zellseparatoren. - Ergebnisse einer retro-

spektiven multizentrischen Studie 7

Böhm, R. und Borberg, H., Köln

Untersuchungen zur Sicherheit der apparativen Zyt-

apherese 18

Höcker, P. und Wagner, A., Wien/Österreich Nebenwirkungen von Zytapheresen mit

Zellseparatoren . 31

Sonderbände 35

Basler, H.D.; Steinfelder, C; Hartnack, U. und Kretschmer, V., Marburg

Sicherheit und Belastung der Spender während der

Thrombozytapherese mit dem Zellseparator. - Eine

psychologische Studie 36

Neumann, H.-J.; Meisberger, A. und Mathieu, B., St.Wendel

Verbesserung der Sicherheitssysteme bei Zellseparato

ren. - Das neue Sicherheitskonzept für den Zellseparator

AS404 (Fresenius) 43

Sniecinski, I.J., Duarte, Calif. I USA 
Sicherheit der Apheresespende

Hämapheresekommission der Deutschen Gesellschaft für

Transfusionsmedizin und Immunhämatologie

Durchführung apparativer Hämapheresen zur Gewinnung von Blutbestandteilkonserven. - Empfehlungen der

Hämapheresekommission der Deutschen Gesellschaft für

Transfusionsmedizin und Immunhämatologie 57

Supplement 5, Oktober 1987

Giovannetti, S., Pisalltalien

Compliance und Ernährungszustand von Patienten unter

einer eiweißarmen, mit Amino-/Ketosäuren supplemen-

tierten Diät 4

Bergström, /.; Ahlberg, M. und

Alvestrand, A., StockholmlSchweden; Fürst, P., Stuttgart

Aminosäurentherapie bei Patienten mit chronischem

Nierenversagen

8

Barsotti, G.; Navalesi, R.; Morelli, E.; Giampietro, O.; Ciardella, F.; Cupisti, A. und Giovannetti, S., Pisalltalien Einfluß einer phosphat- und eiweißarmen Diät, supple-mentiert mit essentiellen Aminosäuren und Ketoanaloga auf die fortgeschrittene diabetische Nephropathie .... 12

Walser, M.; LaFrance, N.; Ward, L.

und VanDuyn, Baltimore/USA

Die Progression chronischer Niereninsuffizienz nach der

Gabe von Ketosäuren und Aminosäuren 17

Gretz, N.; Meisinger, E. und Strauch, M., Mannheim Der Einfluß von Diät und renaler

Grunderkrankung auf die Progression der chronischen Niereninsuffizienz . ... 21

Meisinger, E.; Gretz, N. und Strauch, M., Mannheim

Hyperfiltration unter eiweißarmer Diät induziert durch

Amino/Ketosäuren-Supplemente: Einfluß auf die Pro-

teinurie . 26

Gretz, N.; Meisinger, E. und Strauch, M., Mannheim

Hyperfiltration unter eiweißarmer Diät induziert durch

Amino/Ketosäuren-Supplemente: Einfluß auf die Kreati-

nin-Clearance 30

Sonderbände 33

Schmicker, R., Rostock/DDR; Vetter, K.; Líndenau, K.

und Fröhling, P. T., Potsdam/DDR;

Kokot, F, Katowitz/Polen

Konservative Langzeitbehandlung bei chronischer

Niereninsuffizienz mit Ketosäuren- und Aminosäuren-

substitution 34

Lindenau, K.; Vetter, K. und

Fröhling, P. T., Potsdam/DDR;

Kokot, F., Katowitz/Polen; Schmicker, R., Rostock/DDR

Einfluß von Ketoanalogen auf die renale Osteopathie . . 40

13

Gerok, W., Freiburg Hepatische Enzephalopathien. 
Schomerus, H., Rotenburg (Wümme)

50

Das Krankheitsbild der hepatischen Enzephalopathie

Egberts, E.-H., Tubingen

Therapeutische Studien mit verzweigtkettigen Aminosäu

ren bei portosystemischer Enzephalopathie 53

Schwartz, S.; Farriol, M.; Rodriguez, R.; Garcia, E.;

Padró, J.B. und Vente, P.E., Barcelona/Spanien

Einfluß der qualitativen Kalorienzusammensetzung ente-

raler Ernährungsformeln auf die hepatische Proteinsyn-

these bei normalen und operierten Ratten

59

Schauder, P., Göttingen

Bedeutung der verzweigtkettigen Ketosäuren für den

Proteinstoffwechsel 62 